\title{
Genes, Brains, and Language: An Epistemological Examination of How Genes Can Underlie Human Cognitive Behavior
}

\author{
Nathalie Gontier \\ Konrad Lorenz Institute for Evolution and Cognition Research and Vrije Universiteit Brussel
}

\begin{abstract}
How do genes encode for the formation of morphological structures such as the brain? Can genetic material also encode for behavior such as cognition, language, or culture? For many years, evolutionary biologists as well as scholars who work within extrabiological fields such as psychology, linguistics, and archaeology could only answer the above two questions in a speculative manner. This is because until recently, empirical observations on how genes underlie anatomy or behavior were generally lacking. This situation has now changed. Several genes (MCPH1-MCPH6) have been implicated in the regulation of brain size and a first gene (the FOXP2 gene) has been identified that might underlie linguistic behavior. These discoveries allow us to finally test some of the long-standing theoretical assumptions on how genes do or do not determine morphology and behavior.
\end{abstract}

Keywords: $M C P H$ genes, FOXP2 gene, evolutionary linguistics, psychology, archaeology and epistemology

There is an increasing tendency today to formulate and interpret "classic" linguistic, psychological, and cultural research questions and data of the social and human sciences in the vocabulary of evolutionary biology. Many new academic disciplines have arisen as a consequence of this "naturalistic turn" (Callebaut, 1993). Examples are evolutionary psychology, neural Darwinism, evolutionary linguistics, and evolutionary archaeology. These newly evolving multidisciplinary fields typically borrow domain-specific scientific concepts and methodologies from a variety of academic disciplines to tackle specific problems. Such conceptual hybridization is necessary if we ever want to un-

Nathalie Gontier, Konrad Lorenz Institute for Evolution and Cognition Research, Altenberg, Austria and Centre for Logic and Philosophy of Science, Vrije Universiteit Brussel, Brussels, Belgium.

Warm thanks to John Hunter for all his efforts in both the conference and this volume. Sincere thanks to Patrick Evans for electronically discussing some of the findings concerning brain size genes with me. Research for this article was enabled by a grant provided by the Fund for Scientific Research Flanders, Belgium, while the author was affiliated to the Centre for Logic and Philosophy of Science of the Vrije Universiteit Brussel, Belgium.

Correspondence concerning this article should be addressed to Nathalie Gontier, Vrije Universiteit Brussel, Pleinlaan 2, 1050 Brussels, Belgium. E-mail: nathalie.gontier@vub.ac.be ravel the evolution of language, culture, or the human mind (Gontier, 2006b). Unfortunately, the blending of scientific jargon creates many misinterpretations and has terminological confusions as a consequence. This is especially the case when these scientists investigate how a certain humanspecific cultural and cognitive behavior, such as the capacity to symbolize, is genetically underpinned.

In this article, the assumptions concerning the role of genes in human symbolic behavior that are used in the fields of evolutionary psychology, evolutionary linguistics, and evolutionary archaeology are examined more closely. These ideas are subsequently tested in light of the newly discovered FOXP2, MCPH1, and ASPM genes. Finally, these findings are interpreted from within current evolutionary biological considerations concerning the nature of genetic material.

\section{A (Group of) Gene(s) for All S/Reasons}

Even though ontogenetic or cultural factors are taken into account, it is a commonplace idea within both traditional and evolutionary linguistics, psychology, and archaeology to assume that human symbolic behavior, such as language (Chomsky, 1959, 1965; Pinker, 2001; Pinker \& Bloom, 1990), Theory of Mind and intentional- 
ity (Tomasello, 2004, pp. 18-19), or culture (Klein, 1999; Mellars \& Stringer, 1989) is ultimately genetically determined. The main discussion appears to revolve around the number of genes that underlie these behaviors (one or a multitude) and the pace at which they evolve. More specifically, the idea of genetic determination of cognitive behavior usually takes on the form of a macromutational or micromutational scenario. In the former, a single gene causes a macroevolutionary event that at once leads to the evolutionary emergence of a variety of qualitatively distinct human behaviors (perhaps because the gene underlies a massive cognitive reorganization of the soft brain tissue). In the latter, a series of gradually evolving genes accumulatively encode for human-specific symbolic behavior.

Most of the theories that postulate the existence of currently unidentified genes that encode for human-specific symbolic behavior were formulated when sufficient knowledge of genetic operations was lacking; or before several genes such as the FOXP2 gene or $M C P H 1$ and $A S P M$ were identified and correlated to human-specific behavioral traits. These new discoveries now allow us to test the predictions and theoretical assumptions that were made concerning the role of genes in the evolution of the human brain and its cognitive functions, including language and culture. In what follows, we examine how these recent discoveries shed new light upon the problem of genetic determination of cognitive behavior and how this necessitates the revision of old theoretical assumptions on the subject.

\section{The FOXP2, MCPH1, and ASPM Genes}

We are accustomed to look upon genes as entities that encode for certain morphological features. Although many questions are still left unanswered, we know that codons encode for amino acids that form proteins that in turn form cells and tissue. These tissues and cells ultimately underlie morphological forms. It has also been postulated that genes not only encode for morphological forms, but also for behavior such as language, cognition, or culture. Nevertheless, how precisely genes can underlie such behavior was largely unknown. The recent discovery of the FOXP2, MCPH1, and ASPM genes has changed this situation. Now, earlier speculations concerning the nature of "behavioral" genes can finally be put to the test.

\section{The FOXP2 Gene and Language}

Six years ago, within members of a British family (dubbed the KE-family), a genetic deficiency was unequivocally coupled to their speech and language pathology. In their excitement, media and scientists alike called this gene "the" (or "one of the") "language," "grammar," or "speech" gene(s). But, how is the gene looked upon today? In what follows, the discovery of the gene is reviewed. Subsequently, the workings of the mutated and normal variants of the gene are investigated from within ontogeny and phylogeny.

Half of the members of the KE family suffer from a severe speech deficiency that is diagnosed as verbal dyspraxia, "an impaired ability to perform the coordinated movements that are required for speech" (Vargha-Khadem et al., 2005 , p. 131). Verbal dyspraxia is a quite common language pathology that sometimes arises after a stroke or brain lesions, but it was demonstrated that in the KE family, this deficiency was hereditary and thus caused by a genetic deficiency. Lai et al. $(2000,2001)$ identified the gene that is presumed responsible for the deficit. This gene goes by the name of FOXP2 and is located on the long arm of chromosome 7 (at position $7 \mathrm{q} 31$ ).

FOXP2 stands for FORKHEAD BOX P2. The gene is thus part of a larger, related class of genes called FORKHEAD genes or FOX genes. All FOX genes are regulators of embryogenesis. They contain a forkhead DNA sequence that encodes for a transcription factor that has a specific protein structure that is called the wingedhelix. The winged-helix (or forkhead protein, FOX protein) regulates the transcription of target genes (Vargha-Khadem et al., 2005, p. 133). In other words, FOX genes function in a regulative way because they encode for helix-turn-helix proteins that rather than form tissue, regulate the (dis)activation and structure of other genes (Carlsson \& Mahlapuu, 2002, p. 1; VarghaKhadem et al., 2005, pp. 132-134). The FOXP2 protein in particular "interacts with the regulatory regions of downstream target genes and controls their expression by repressing the level and/or rate of transcription" (Vargha-Khadem et al., 2005, p. 134). Thus, the FOXP2 protein 
can switch other genes on or off or "modulate the expression of other genes" (Fisher, 2006, p. 287). The affected KE family members have a single amino acid missense mutation in their FOXP2 protein and this substitution most probably leads to a loss of function of one copy of the FOXP2 gene (Vargha-Khadem et al., 2005, p. 133). In summary, for the first time in history, an identified genetic deficiency can be associated with a speech pathology. This has made scholars postulate that the normal variant of the FOXP2 gene is implicated in normal language development and ultimately language evolution (Lai et al., 2003; Marcus \& Fisher, 2003).

However, two observations make the position that the FOXP2 gene is (one of the) long searched for "human language" gene(s) highly problematic. First, scholars cannot yet explain what exactly the mutated form of the gene is causing to go wrong. They are currently unable to demarcate the core deficit that the affected KE family members suffer from. Their pathology has been characterized more narrowly as verbal dyspraxia (Hurst et al., 1990), or more broadly as Specific Language Impairment (Gopik, 1990). That mutations in the FOXP2 gene are somehow related to verbal and orofacial dyspraxia has subsequently been confirmed by several different studies, including genetic studies in individuals who are unrelated to the KE-family and who suffer from different mutations in their FOXP2 gene (Feuk et al., 2006; MacDermot et al., 2005; Zeesman et al., 2006). These studies therefore also lend credibility to the idea that the normal variant of the gene is involved in orofacial skills that are required for articulate speech.

However, the deficit of the affected KEfamily members is sometimes also diagnosed as Specific Language Impairment because the patients have not only impaired language performance skills, but also impaired language competence. More specifically, Gopnik (1990) pointed out that the affected KE family members demonstrated selective grammatical deficits, especially regarding the use of inflections. She therefore speculated that grammar might be genetically underpinned and that a deficit in such a "grammar" gene was responsible for the deficiency. The results of the Gopnik-study were partly confirmed and partly falsified by experiments conducted by Vargha-Khadem et al. (1995, p. 932). These scholars indeed found grammatical deficits to be present in the behavioral phenotype of the affected family members, but they were also able to distinguish a series of other linguistic deficiencies (regarding lexical decisions, picture vocabulary, writing, rhyme production, etc.). They therefore conclude that "The evidence from the KE family thus provides no support for the proposed existence of grammar-specific genes" (VarghaKhadem et al., 1995, p. 930). However, the affected KE family members also deviate in more than one way from the classical symptoms of SLI. To begin with, other SLI patients mostly do not suffer from a mutation in their FOXP2 gene. Other chromosomes (especially chromosome 13, 16, and 19) are implicated in SLI (Bishop, 2002a, p. 312, 2002b, 2003, pp. 315-8; Fisher \& Marcus, 2006, p. 14). Moreover, the affected KE family members score slightly lower than average on nonverbal IQ-tests (Vargha-Khadem et al., 1995), and they portray a variety of structural and functional brain abnormalities (Liégeois, 2003; Vargha-Khadem et al., $1995,1998,2005)$. These characteristics normally falsify the diagnosis of SLI (Bishop, 2003).

A second observation that demonstrates the need for caution in denoting the FOXP2 gene as a "human-specific language" gene is the following. Orthologs of the gene can be found that predate the origin of our species; the normal human-specific variant of the gene is also found in Neanderthal-DNA (Krause et al. 2007); and in humans, the gene is not exclusively active in language-related structures such as the brain. It is widely accepted that language is a humanspecific trait. Many scholars have therefore traditionally assumed that if language is genetically underpinned, these "language genes" must either have evolved de novo in our species, or, if they are present in other species, must have undergone human-specific mutations. Moreover, it is reasonable to assume that, given the enormous amount of things such "language genes" must encode for, they would be exclusively involved in the formation of languagerelated structures. It has traditionally been assumed that a gene for language will not simultaneously be implicated in the formation of other morphological or behavioral structures. Incoming results of the ontogenetic activation patterns of the gene as well as the phylogenetic evolution of the gene, however, make all these classic theoretical assumptions untenable. 
To begin with, the FOXP2 gene is not exclusively human. Orthologs of the gene, as well as the gene group of which it is a part (i.e., the FOX genes), can be found in most multicellular organisms from the origin of fungi onward (Carlsson \& Mahlapuu, 2002, pp. 1). Thus, they are very old, and their Forkhead Box Domain (that encodes for the proteins) is highly conserved in evolution. This high level of conservation indicates that these genes are probably the target of positive selection (Carlsson \& Mahlapuu, 2002, p. 24). Two independent studies (Enard, 2002, p. 869; Zhang, Webb, \& Podlaha, 2002, p. 1829) have demonstrated that also the FOXP2/FoxP2 protein in particular has been the target of positive selection. Enard et al. (2002, p. 870) has demonstrated that since the split with the mouse (which happened 70 million years ago) only three (functional) changes in amino acid sequences have occurred in the Foxp2/Foxp2 protein. Two of these changes occurred solely within the human lineage and these changes appear to be fixed in the population (i.e., few polymorphisms can be found although some variation has been reported). Zhang, Webb, \& Podlaha (2002, p. 1829) have however demonstrated that one of these substitutions has also occurred within carnivores. Because carnivores do not posses anything like human language, this substitution cannot account for the rise of language.

The human-specific amino acid substitutions are calculated to have occurred around 200,000 years ago (Enard et al., 2002, p. 870). This date converges with the rise of anatomically modern humans. Many evolutionary archaeologists, psychologists, and linguists exclusively attribute the capacity for language to this variant of the human species. Therefore, it has been suggested that the specific amino acid substitutions might be responsible for the rise of fully modern speech. However, this view has been questioned since the date for the human-specific amino acid substitutions is currently being pushed further back in time.

Furthermore, the regions where FOX/Fox genes in general and the FOXP2/Foxp 2 gene in particular are expressed are currently being mapped. Incoming results demonstrate that they are not exclusively active in (homolog) language-related brain areas. In vertebrate development, Fox genes in general are active in both embryogenesis and neurogenesis (Carlsson \&
Mahlapuu, 2002, pp. 10-15) and they appear to correlate with the complexity of the body plan. Drosophila (the fruit fly) has 20 Fox genes while Homo sapiens has 39 FOX genes (Carlsson \& Mahlapuu, 2002, pp. 1-23). The activation pattern of the Foxp/FoxP genes in particular has been mapped for zebrafish (Bonkowsky \& Chien, 2005; Shah et al., 2006; Tamura et al., 2003), the mouse (Ferland et al., 2003; Takhahashi et al., 2003; White et al., 2006), and zebra finches (a songbird, Haesler et al., 2004; Teramitsu et al., 2004). In all these organisms, the Foxp genes are especially active in the brain, especially in motor-related areas and these activation patterns highly resemble those of human FOXP genes.

Turning specifically to the Foxp2 gene of mice, Shu et al. (2005) have studied mice that were specifically bred to have a disruption in either one or both copies of their Foxp2 gene. The results demonstrate that when both copies of their Foxp2 gene are disrupted, the mice suffer from severe motor impairments and die prematurely. However, most importantly, they do not utter ultrasonic vocalizations when they are taken away from their mothers, while normal mice infants always make such vocalizations. The ultrasonic vocalizations remained present when a single copy of the gene was disrupted, but were highly altered. Furthermore, learning and memory appear to be unaffected when only one copy of the gene is disrupted, but a slight developmental delay can be detected. Finally, the cerebellum of all mice developed abnormally. The authors therefore conclude that "the transcription factor Foxp2 [...] may play an important role in cerebellar development and vocalization" (Shu et al., 2005, p. 9648).

In zebra finches, the neural expression of the FoxP2 gene highly increases in males during the critical period in which they learn to sing (Haesler et al., 2004; Teramitsu et al., 2004). In addition, these expression patterns are highly similar to the neural expression patterns of the human FOXP2 gene. The increased activation is not detected in female finches who do not learn to sing.

In summary, the mice and songbird studies mentioned above relate orthologs of the gene to vocalizations. Those brain regions of mice, songbirds, and zebrafish wherein the protein is active, form the human counterparts (or homologs) of language-related brain areas. Nonetheless, although the gene and its protein is 
related to human speech, animal vocalizations, and several brain regions that are implicated in language, it is also active in other regions of the CNS and during other phases of embryogenesis (Fischer, 2006, p. 288). Therefore, it can neither be characterized as an exclusive "human language gene" nor for that matter, as a "bird song gene," "mouse ultrasonic vocalization gene," "motor gene," "putamen gene," "cerebellum gene," and so forth. That is, it cannot actually carry in its structure the information on the formation of all these different structures. If it could carry this amount of information, it would indeed be a "super gene." The discovery of the FOXP2 gene has therefore firmly questioned all present theories on language evolution and how the language activity can be encoded for in our genome.

\section{The MCPH1 and ASPM Genes and the Human Brain}

During the last two million years, hominin brains almost doubled in size from $800 \mathrm{~cm}^{3}$ for Homo ergaster to $1,450 \mathrm{~cm}^{3}$ (on average) for Homo sapiens (Holloway, 1999). On the other hand, from seven to two million years ago, the brains of early hominins do not deviate that much from the brain size of the great apes (Gontier, 2008, pp. 185-217). How are we to explain the steady and gradual evolution of brain size for five million years and the rapid increase in brain size during the past two million years? In evolutionary linguistics, psychology, and archaeology (Klein, 1999; McBreaty \& Brooks, 2000; Mellars \& Stringer, 1989), the sudden increase in brain size that occurred from Homo ergaster onward is assumed to hint at increased cognitive abilities underlying new types of ecological, social, and cultural behavior. Brain expansion, together with a possible reorganization of the soft brain tissue, supposedly enabled the rise of modern human behavior and again, single or multiple genes are held responsible for these changes.

The study of a human pathology called congenital microcephaly now allows us to test these hypotheses because the disease sheds light on the form that such "brain size genes" might take on. Congenital microcephaly is a condition in which the human brain is largely reduced in size. Microcephalics have a head circumference of roughly 40 to $45 \mathrm{~cm}$ as opposed to the normal 53 to $59 \mathrm{~cm}$. This results in a brain size of about 400 $\mathrm{cm}^{3}$, which is equivalent to the brain size of the great apes or early australopiths (Gilbert, Dobyns, \& Lahn, 2005, p. 585). Depending on the type of microcephalia, several genetic deficiencies (located on different chromosomes) have been correlated to the disease (Gilbert, Dobyns, \& Lahn, 2005, pp. 584-586; Evans et al., 2005, p. 1717; Evans, Vallender, \& Lahn, 2006). All these genes are especially active during neurogenesis, either during the proliferation or differentiation of the neuroblasts or neural stem cells, or within the mitotic spindle. Of these genes, MCPHI (Microcephalin 1) and ASPM (Abnormal Spindle-like Primary Microcephaly associated) are the most well-studied and it are these genes that are regarded as developmental regulators of brain size.

Phylogenetically, the MCPHI gene was highly selected for during the evolution from the simians to the great apes. ASPM has been the target of positive selection during the evolution from the great apes to humans. Polygenetic analyses of extant humans also demonstrate ongoing positive selection for these genes within our species (Evans et al., 2005, p. 1717; for a critique see Currat et al., 2006). More specifically, Gilbert, Dobyns, \& Lahn (2005, p. 586) demonstrate that the $M C P H 1$ and $A S P M$ genes have undergone an accelerated evolution within a specific haplotype group called haplotype D, the D stands for derived (Evans et al., 2005 , p. 1718). One human-specific variant of the haplogroup D of the $M C P H 1$ gene is estimated to be 37,000 years old, while the coalescence age ("the time to the most recent common ancestor," Mekel-Bobrov et al., 2005, p. 1722) for the rest of the chromosomes under investigation goes back 1,700,000 years ago (Evans et al., 2005, p. 1718). Because anatomically modern humans are estimated to be 200,000 years old, this variant of the haplogroup D of the $\mathrm{MCPHI}$ gene is thus younger than our species' origin. ${ }^{1}$ In other words, since our species' origin, this region of the gene has continued to evolve, presumably because it remains the target of positive selection. One human-specific variant of the haplogroup D of the ASPM gene is estimated to be 5,800 years old, while the coalescence time of the rest of the chromosomes

\footnotetext{
${ }^{1}$ Later simulations conducted by Evans et al. (2006) also make it plausible that at least the haplogroup D of $\mathrm{MCPHI}$ might have originated much earlier in another Homo lineage and introgressed in our species due to hybridization with other Homo species.
} 
is estimated at 800,000 years (Mekel-Bobrov et al., 2005, p. 1720). In addition, this variant too has seen a fast rise in our species since its origin.

Why these genes continue to be positively selected is unknown. Concerning the specific function of MCPH1, Evans et al. (2005, p. 1720) assert: "The specific function of Microcephalin in brain development makes it likely that selection operated on the brain. Yet, it remains formally possible that an unrecognized function of Microcephalin outside of the brain is actually the substrata of selection." That the genes were positively selected for their role in brain size regulation has been criticized by Woods et al. (2006, pp. 2025-2026). They investigated the regular variants of $M C P H I$ and $A S P M$ of 120 individuals with no known deficiencies, and also measured their brain size with the help of MRI scans. This team finds no evidence that the selected alleles are associated with an increase or decrease in brain size during ontogeny. Phylogenetically, they notice that the brain size of Homo sapiens has not been increasing during the last 50,000 years; rather it has been decreasing in size (Woods et al., 2006, p. 2027). This causes problems for the statement that these genes are responsible for the increase in brain size in our species, since they are dated younger than 50,000 years.

Moreover, Woods et al. (2006, p. 2027) note that ASPM and MCPHI both are also expressed outside the brain, during immune defenses, sensory perception, tumor suppression, apoptosis, and spermatogenesis. This makes it possible that, in the end, these genes do not actually have anything to do with an increase in brain size. Rather, their reason for being positively selected might lie in these nonneurological activation patterns.

In the original papers, the members of Lahn's team noticed that the estimated date of 37,000 years for the $M C P H I$ variant correlates with the Upper Palaeolithic "symbolic revolution," whereas the ASPM variant, dated to be 5,800 years old, correlates with the rise of cities and writing. The authors emphasize that it is currently unknown whether these correlations are significant. Nonetheless, pointing out such correlations can be potentially misleading. Since the original papers, the rise of the MCPH1 variant has now been dated to 1.1 million years ago, and this undoes the correlation with the rise of the "symbolic revolution" (estimated at 50,000 years ago, if there ever was such a thing, see, e.g., McBreaty and Brooks for a critical appraisal of the idea). 1.1 million years ago, our species had not yet evolved and the then living archaic Homo species presumably did not produce cave paintings that are associated with later Upper Palaeolithic art industries.

In summary, several genetic variations within the human species have been linked with microcephalia and hence with brain size. Especially the $M C P H 1$ and $A S P M$ genes have undergone positive selection for many millions of years. Some of the human-specific variants of these genes have evolved fairly recently, even after the origin of anatomically modern species. Thus, these human-specific variants currently continue to be positively selected within the human species. These results make it tempting to conclude that these genes are actually "brain size regulating genes," that the regulation of brain size is the exclusive function of these genes, and that this is the reason why they evolved and continue to be the subject of positive selection. The genes involved, however, cannot straightforwardly be identified as such "brain size genes" because, besides their apparent involvement in brain size regulation, these regulatory genes are also active outside the brain in a variety of morphological structures.

Should we then regard these genes as "microcephalic genes" as well as "neurogenesis genes," "neural stem cell genes," "immune defense genes," "sensory perception genes," "apoptosis genes," "spermatogenesis genes," "genes that enable us to live in cities," "symbolic revolution genes," and so forth? In addition, if so, should we then also be looking for commonalities between these various traits? Should we be investigating the relation between brain size and spermatogenesis; or the relation between immune defenses and symbolic behavior? On the other hand, is the fact that these genes are implicated in all these features a mere coincidence, without these features having anything in common? Moreover, given this variety of functions that the genes are involved in, should we measure which functions provide the most survival benefit and only recognize the most beneficial function as the exclusive target of positive selection? At present, we cannot fit the results into our theoretical frameworks. 


\section{Preliminary Conclusion}

The incoming results from the study of the FOXP2 gene and MCPHI-ASPM genes are thoroughly shaking our conceptions on how genes can encode for a certain physical or behavioral trait. More often than not, the incoming data do not fit our theoretical frameworks and rather than provide answers, they leave us with a series of new methodological questions concerning the nature of genes. The classic theoretical assumptions that assumed that genes can unequivocally be coupled to a certain function (e.g., the idea that "x is a gene for y") do not appear to be adequate to interpret the incoming results. In other words, we cannot understand the $M C P H 1$ or $A S P M$ gene as exclusive "brain size genes" or the FOXP2 gene as an exclusive "language gene." Rather, in both cases, the question arises how it is possible that one and the same gene can be active during the formation of several distinct morphological structures and still be implicated in brain morphology or language and hence possibly also in cognitive, symbolic behavior. This last question in turn requires an answer that highlights current epistemological shifts concerning the nature of genes in evolutionary and molecular biology.

\section{Paradigm Shifts Concerning the Nature of Genes in Evolutionary Biology}

Even before the genetic code was cracked, evolutionary biologists speculated on the nature of genes (Schwartz, 1999). The idea that there exist hereditary particles that somehow underlie morphological traits was first introduced by Mendel in the 19th century. He argued that "factors" are responsible for the variation that can be seen between members of the same species. In the 1930s, population geneticists such as de Vries speculated that changes or "mutations" of "genes" not only underlie variation but that they can actually cause the introduction of novel traits. From then on, genes and especially mutations of these genes were assumed to cause evolution, and this evolution was driven by natural selection. Thus, in the 1930s, Mendel's hereditary laws were combined with de Vries' mutation theory and Darwin's mechanism of natural selection. This is known as the Modern Synthesis and its adherents are called Neodarwinists rather than Darwinists because they adjusted as well as abandoned some of the basic tenets of Darwin's ideas on evolution.

Neodarwinists postulated that genes underlie the formation of (novel) morphological features. Therefore, they had to examine how exactly genes can cause the formation of certain morphological features. They argued that genes inherently carry "information" on how certain traits are formed. The idea that genes carry such information was further intensified when the genetic "code" (as it became called) was "cracked" by Francis Crick and James Watson in 1953. After this, it was assumed that when a gene randomly mutates, its information on morphology-formation either becomes "nonsensical" or the information is altered and new information emerges. When this new morphological structure is adaptive for the carrier, it can become the target of positive selection. Furthermore, genes are argued to always carry this information (inherently or potentially) even if the genes are not phenotypically expressed.

In summary, Neodarwinians are principally interested in the mechanisms by which this genetic information is formed and transmitted to the next generation. Elsewhere (Gontier, 2006a, 2006c), I have already demonstrated that the Neodarwinian emphasis on the "how question," together with the idea that natural selection targets the phenotype, recalls the mechanistic turn of the 19th and early 20th century. This emphasis on mechanism started to change in the 1960s. One of the Neodarwinists, Ernst Mayr, wrote a series of articles in which he defended evolutionary biology as a science that progresses along different lines than physics. According to Mayr (1961, p. 360), evolutionary biology not only asks "how" a trait evolved, it also asks "why" it evolved. The "why" question can be reformulated as "how come," as well as "what for." The former question asks about the mechanism, the latter question asks about the function of a trait. Functional explanations can also lead to teleological explanations (e.g., that the eye evolved for vision). Mayr's articles would inspire a series of debates about whether or not evolutionary biology should refrain from teleological jargon; that is, whether or not it is allowed to ask "what" a trait evolved "for," especially when adaptations are the subject under investigation.

Williams (1966) argued that adaptations deserve to be studied independently, and he 
developed the discipline of teleonomics specifically for this purpose. The latter would evolve into sociobiology and eventually its goals would be incorporated into current evolutionary psychology. Contrary to Mayr and Neodarwinian scholars in general, Williams asserted that the entire genotype (not the phenotype alone) is the unit of selection and thus that also noncoding genes could become the target of selection. Both points were further elaborated upon by Richard Dawkins (1983). He too argued that genes rather than the phenotype are the unit of selection and that evolutionary biology should primarily be concerned with the explanation of adaptations, that is, morphological forms that appear "as if" they have been "designed" for the "function" or "purpose" they perform (Dawkins, 1983, p. 16). Both Williams and Dawkins are therefore called Post-Neodarwinists (Schwartz, 1999), because, contrary to Neodarwinian scholars, they introduce functionalist approaches into evolutionary biology.

The difference between Neodarwinians and Post-Neodarwinians is best explained by an example such as the evolution of the eye. Neodarwinians mainly focus on how the morphological structure of the eye could be coded for in our genes and become phenotypically expressed. Post-Neodarwinians further argue that the function of the eye (i.e., vision) is important for its future selection and evolution. Computer simulations (Dawkins, 1995) have demonstrated that it would "only" require 400,000 generations to gradually evolve an eye by means of natural selection. However, such a scenario also requires the presence of intermediate organisms. Critics (e.g., Schwartz, 1999, 361-362) have therefore asked what the survival benefit might be of an "intermediate" organism with only 5\% of an eye. Dawkins's reply is that these intermediate, "unfinished" structures are preadaptations of vision: even $5 \%$ of vision is better than no vision. During the course of evolution, selection will naturally favor organisms with better vision and this will result in an "end product" that appears "as if" it was designed for its current function. Such "accumulative selection"-as Dawkins (1983) calls it-where nonoptimally functioning traits are replaced by a series of intermediate organisms with everbetter functioning traits, can only occur when genes inherently carry their information. That is, the evolution toward information on how to built a functional eye cannot emerge anew in every one of the 399,000 generations that precede a fully functional eye. Rather the information accumulates and is thus somehow retained in the genome.

In summary, both Neodarwinian as well as Post-Neodarwinian scholars endorse the view that genes encode for information. Genes carry information about the form of a trait (according to the Neodarwinians), or information about the form as well as the function of a trait (according to Post-Neodarwinians). This inherent information is argued to be transmitted from one generation through the next via the reproduction process. Because of new data on the workings of genetic material, this information-transmission metaphor that is typical of both schools of thought is currently being replaced by what can be characterized as an information-emerging metaphor. The latter metaphor is especially applied in system theory (see also Shanker \& King, 2002).

Within systems theory, genes are not understood as particles that actually encode for specific anatomical traits or behavioral features. Rather than assuming that information is inherently present in genes and faithfully transmitted, information is understood as an exherent, emerging property of genes. It is the interactions between different genes (through the proteins they encode for) and the interactions between genes and the environment that will result in the formation of certain structures. The roots of this view lie with Schrödinger who, at the beginning of the 20th century, already argued that genes underlie "difference in properties" rather than they encode for properties (Schrödinger, 2000, pp. 28-29). It is impossible to reduce the formation of a physical trait or the development of a certain behavior to a single gene or a group of genes because at each step during development, a variety of factors need to be taken into account that surpass the workings of genes and gene-products. At best, genes encode for amino acids that in certain circumstances form proteins or enzymes, and so forth. The latter, in turn, form cells that under specific circumstances lie at the basis of tissue formation and so on.

Interestingly, both theoretical frameworks (the information-transmission metaphor as well as the information emerging metaphor) hold true to a certain extent. Molecular geneticists 
and evolutionary biologists currently divide genes into structural and regulatory genes. Structural genes are genes that are assumed to encode for a certain morphological trait. In other words, here it is assumed that there exists a one-to-one correspondence between a trait and the transcription of a specific gene into proteins, and so forth. Regulatory genes, on the contrary, are genes that cannot straightforwardly be associated with the rise of a certain morphological trait. The proteins that these genes encode for do not form tissue but orchestrate the overall activation and deactivation of other genes and as such are responsible for the body plan of an organism. For example, the same gene that makes humans bipedal organisms, makes sea stars radially symmetrical (Schwartz, 1999). Gehring (1998) therefore calls these genes "master control genes of development." In addition, the above described genes are part of the latter group.

Regulatory and structural genes are not mutually exclusive. Both are involved in the evolution of life. In the eye for example, it appears that structural genes underlie eye color, while regulatory genes underlie the actual formation of the eye. Gehring (1998) discovered that one regulatory gene can turn on 2,500 genes that are involved in eye formation. Neither this single gene nor the other genes that it activates inherently carry information on how to form an eye. Rather, this information emerges when these different genes start interacting in a certain manner, at a certain time and at a certain locus. In sum, the information to build an eye emerges during heterochronic processes.

\section{Conclusions}

Given the incoming results on the FOXP2 gene as well as the ASPM and MCPHI genes, together with the described shift from an information-transmission to an information-emerging metaphor in genetics makes us raise the following question: How do these findings affect old, theoretical assumptions that there exist such things as "language genes" or "brain size genes"? The above described "brain size genes" as well as the FOXP2 gene (and the larger class of FOX genes where it is part of) are all regulatory genes. Regulatory genes cannot straightforwardly be related to morphological, let alone behavioral, features of an organism. That is, these regulatory genes do not inherently carry the information to built larger brains, or language modules. Rather, such morphological and behavioral features emerge due to the genes that these regulatory genes (de)activate via the proteins they encode for; in combination with numerous other environmental factors. Specifically regarding the brain, Fisher (2006, p. 270) for example argues that " $[\mathrm{g}]$ enes do not specify behaviors or cognitive processes; they make regulatory factors, signaling molecules, receptors, enzymes, and so on, that interact in highly complex networks, modulated by environmental influences, in order to build and maintain the brain."

The shift from an information-transmission to an information-emerging metaphor concerning the nature of genetic material has not yet reached new disciplines such as evolutionary linguistics, psychology, or archaeology. It is curious how, at a time when evolutionary biologists and molecular geneticists are abandoning the "blueprint" metaphor and are focusing on gene-protein interactions and epigenetics, the new evolutionary sciences are more than ever subscribing to gene-deterministic views. It has become fashionable to assume that every human-specific cognitive behavior (language, speech, grammar, culture, imitation, etc.) is ultimately determined by one or a few genes. The new data on the nature of genes however, make a lot of the classic, theoretical assumptions on the existence of symbolic, language, cultural or cognitive genes untenable. It will be a challenge for the future to reformulate the current theoretical assumptions in light of these new discoveries.

\section{References}

Bishop, D. V. M. (2002a). The role of genes in the aetiology of specific language impairment. Journal of Communication Disorders, 35, 311-328.

Bishop, D. V. M. (2002b). Putting language genes in perspective. Trends in Cognitive Sciences, 18, 57-59.

Bishop, D. V. M. (2003). A comparison of language abilities in adolescents with Down Syndrome and children with specific language impairment. Journal of Speech, Language, and Hearing Research, 46, 1324-1339.

Bonkowsky, J. L., \& Chien, C. (2005). Molecular cloning and developmental expression of foxP2 in 
Zebrafish. Developmental Dynamics, 234, 740-746.

Callebaut, W. (1993). Taking the naturalistic turn or how real philosophy of science is done. Chicago: The University of Chicago Press.

Carlsson, P., \& Mahlapuu, P. (2002). Forkhead transcription factors: Key players in development and metabolism. Developmental Biology, 250, 1-23.

Chomsky, N. (1959). A review of B. F. Skinner's verbal behaviour. Language, 35, 26-58.

Chomsky, N. (1965). Aspects of the theory of syntax. Cambridge, MA: The MIT Press.

Currat, M., et al. (2006). Comment on 'Ongoing adaptive evolution of ASPM, a brain size determinant in Homo sapiens' and 'Microcephalin, a gene regulating brain size, continues to evolve adaptively in humans. Science, 313, 172a.

Dawkins, R. (1983). Universal Darwinism. In D. Hull \& M. Ruse (Eds.). (1998), The philosophy of biology (pp. 15-35). New York: Oxford University Press.

Dawkins, R. (1995). River out of Eden. San Francisco: Harper Collins.

Enard, W., et al. (2002). Molecular evolution of $F O X P 2$, a gene involved in speech and language. Nature, 418, 869-872.

Evans, P., et al. (2006). Evidence that the adaptive allele of the brain size gene microcephalin introgressed into Homo sapiens from an archaic Homo lineage. Proceedings of the National Academy of Sciences, USA, 103, 18178-18183.

Evans, P. D., et al. (2005). Microcephalin, a gene regulating brain size, continues to evolve adaptively in humans. Science, 309, 1717-1720.

Evans, P. D., Vallender, E. H., \& Lahn, B. T. (2006). Molecular evolution of the brain size regulator genes CDK5RAP2 and. CENPJ. Gene, 375, 75-79.

Ferland, R. J., et al. (2003). Characterization of Foxp2 and Foxp1 mRNA and protein in the developing and mature brain. The Journal of Comparative Neurology, 460, 266-279.

Feuk, L., et al. (2006). Absence of a paternally inherited FOXP2 gene in developmental verbal dyspraxia. The American Journal of Human Genetics, 79, 965-972.

Fisher, S. E. (2006). Tangled webs: Tracing the connections between genes and cognition. Cognition, 101, 270-297.

Fisher, S. E., \& Marcus, G. F. (2006). The eloquent ape: Genes, brains, and the evolution of language. Nature Reviews, Genetics, 7, 9-20.

Gehring, W. (1998). Master control genes in development and evolution: The homeobox story. London: Yale University Press.

Gilbert, S. L., Dobyns, W. B., \& Lahn, B. (2005). Genetic links between brain development and brain evolution. Nature reviews, genetics, 6, 581-590.
Gontier, N. (2006a). An epistemological inquiry into the "what is language" question and the "what did language evolve for' question. In A. Cangelosi, A. D. M. Smith, \& K. Smith, (Eds.), The evolution of language: Proceedings of the 6th international conference (Evolang 6) (pp. 107-115). London: World Scientific.

Gontier, N. (2006b). Introduction to evolutionary epistemology, language and culture. In N. Gontier, J. P. Van Bendegem, \& D. Aerts (Eds.), Evolutionary epistemology, language and culture (pp. 1-29). Dordrecht: Springer.

Gontier, N. (2006c). Language and pathologies: An epistemological reflection. Cognitive Systems, 7, 33-60.

Gontier, N. (2008). The dynamics of language activity, an evolutionary reconstruction. Unpublished doctoral dissertation, Vrije Universiteit Brussels, Brussels, Belgium.

Gopnik, M. (1990). Feature-blind grammar and dysphasia. Nature, 344, 715.

Haesler, S., et al. (2004). FoxP2 expression in Avian vocal learners and non-learners. The Journal of Neuroscience, 24, 3164-3175.

Holloway, R. L. (1999). Evolution of the human brain. In A. Lock, \& C. Peters (Eds.), Handbook of human symbolic evolution (pp. 74-125). Oxford: Blackwell Publishers.

Hurst, J. A., et al. (1990). An extended family with a dominantly inherited speech disorder. Developmental Medicine and Child Neurology, 32, 352-355.

Klein, R. G. (1999). The human career. Chicago: The University of Chicago Press.

Krause, J., Lalueza-Fox, C., Orlando, L., Enard, W., Green, R. E., Burbano, H. A., et al. (2007). The derived FOXP2 variant of modern humans was shared with Neanderthals. Current Biology, 17, $1908-1912$.

Lai, C. S., et al. (2000). The SPCH1 region on Human 7q31: Genomic characterization of the critical interval and localization of translocations associated with speech and language disorder. American Journal of Human Genetics, 67, 357-368.

Lai, C. S., et al. (2001). A Forkhead-domain gene is mutated in a severe speech and language disorder. Nature, 413, 519-523.

Lai, C. S., et al. (2003). FOXP2 expression during brain development coincides with adult sites of pathology in a severe speech and language disorder. Brain, 126, 2455-2462.

Liégeois, F., et al. (2003). Language fMRI abnormalities associated with $\mathrm{FOXP} 2$ gene mutation. $\mathrm{Na}$ ture Neuroscience, 6, 1230-1237.

MacDermot, K. D., et al. (2005). Identification of FOXP2 truncation as a novel cause of developmental speech and language deficits. American Journal of Human Genetics, 76, 1074-1080. 
Marcus, G., \& Fisher, E. (2003). FOXP2 in focus: What can genes tell us about speech and language? Trends in Cognitive Sciences, 7, 257-262.

Mayr, E. (1961). Cause and effect in biology. In E. Mayr (Ed.), 1997, Evolution and the diversity of life: Selected essays (pp. 359-382). Cambridge, MA: Harvard University Press.

McBreaty, S., \& Brooks, A. (2000). The revolution that wasn't: A new interpretation of the origin of modern human behaviour. Journal of Human Evolution, 39, 453-563.

Mekel-Bobrov, N., et al. (2005). Ongoing adaptive evolution of $A S P M$, a brain size determinant in Homo sapiens. Science, 309, 1720-1722.

Mellars, P. A., \& Stringer, C. (Eds.). (1989). Introduction: The origins and dispersal of modern humans. In P. A. Mellar \& C. Stringer, The human revolution: Behavioural and biological perspectives on the origins of modern humans (pp. 1-14). Princeton: Princeton University Press.

Pinker, S. (2001). Talk of genetics and vice versa. Nature, 413, 465-466.

Pinker, S., \& Bloom, P. (1990). Natural language and natural selection. Behavioural and Bran Sciences, 13, 707-784.

Schrödinger, E. (2000/1944). What is life? Cambridge, MA: Cambridge University Press: Canto.

Schwartz, J. H. (1999). Sudden origins: Fossils, genes, and the emergence of species. New York: Wiley.

Shah, R., et al. (2006). Expression of FoxP2 during zebrafish development and in the adult brain. International Journal of Developmental Biology, 50, 435-438.

Shanker, S., \& King, B. (2002). The emergence of a new paradigm in ape language research. Behavioral and Brain Sciences, 25, 605-656.

Shu, W., et al. (2005). Altered ultrasonic vocalization in mice with a disruption in the Foxp 2 gene. Proceedings of the National Academy of Sciences, USA, 102, 9643-9648.

Takhahashi, K., et al. (2003). Expression of Foxp2, a gene involved in speech and language in the developing and adult stratum. Journal of Neuroscience Research, 73, 61-72.
Tamura, S., et al. (2003). Expression pattern of the winged-helix/forkhead transcription factor FoxP1 in the developing central nervous system. Gene Expression Patterns, 3, 193-197.

Teramitsu, I., et al. (2004). Parallel FoxP1 and FoxP2 expression in songbird and human brain predicts functional interaction. The Journal of Neuroscience, 24, 3152-3163.

Tomasello, M. (2004). The human adaptation for culture. In F. Wuketits \& C. Antweiler (Ed.), Handbook of human evolution (Vol. 1, pp. 1-23). Weinheim: Wiley-VCH Verlag GmbH \& Co.

Vargha-Khadem, F., et al. (1995). Praxis and nonverbal cognitive deficits in a large family with a genetically transmitted speech and language disorder. Proceedings of the National Academy of Science, USA, 92, 930-933.

Vargha-Khadem, F., et al. (1998). Neural basis of an inherited speech and language disorder. Proceedings of the National Academy of Science, USA, 95, 12695-12700.

Vargha-Khadem, F., et al. (2005). FOXP2 and the neuroanatomy of speech and language. Nature Reviews, Neuroscience, 6, 131-138.

White, S. A., et al. (2006). Singing mice, songbirds, and more: Models for FOXP2 function and dysfunction in human speech and language. The Journal of Neuroscience, 26, 10376-10379.

Williams, G. C. (1966). Adaptation and natural selection. Princeton: Princeton University Press.

Woods, R. P., et al. (2006). Normal variants of $M i$ crocephalin and ASPM do not account for brain size variability. Human Molecular Genetics, 15 , 2025-2029.

Zeesman, S. (2006). Speech and language impairment and oromotor dyspraxia due to deletion of $7 \mathrm{q} 31$ that involves FOXP2. American Journal of Medical Genetics, 140A, 509-514.

Zhang, J., Webb, D. M., \& Podlaha, O. (2002). Accelerated protein evolution and origins of human-specific features: FOXP2 as an example. $G e$ netics, 162, 1825-1835.

Received February 1, 2007 Accepted February 1, 2008 